\title{
CYLINDRICAL HIERARCHY FOR DEFORMING NECKLACES
}

\author{
SERGEY BEREG \\ Department of Computer Science, University of Texas at Dallas, \\ Box 830688, Richardson, TX 75083, USA. E-mail: besp@utdallas.edu \\ Received (received date) \\ Revised (revised date) \\ Communicated by (Name)
}

\begin{abstract}
Recently, Guibas et al. ${ }^{10}$ studied deformable necklaces - flexible chains of balls, called beads, in which only adjacent balls can intersect. In this paper, we investigate the problem of covering a necklace by cylinders. We consider several problems under different optimization criteria. We show that optimal cylindrical cover of a necklace with $n$ beads in $\mathbb{R}^{3}$ by $k$ cylinders can be computed in polynomial time. We also study a bounding volume hierarchy based on cylinders.

Keywords: Bounding Volume Hierarchy; Collision Detection; Smallest Enclosing Cylinder.
\end{abstract}

\section{Introduction}

Our study is motivated by the representation and manipulation of molecular configurations, modeled by a collection of spheres. The representation of threedimensional geometric structure of a molecule with spheres where each atom is viewed as a rigid sphere is a common approach. The sizes of spheres depend on the atom types. There are recommended values for the radius of each atom sphere which is called van der Waals radius. The distance between the centers of every pair of spheres is also known. In this model, the spheres of atoms in a chemical bond interpenetrate. The fused spheres have been studied in computational geometry. Halperin and Overmars ${ }^{11}$ proved useful properties of the spherical model. For example, the combinatorial complexity of the boundary of a molecule is linear.

Recently Guibas et al. ${ }^{10}$ studied the spherical model under motion. They call the spherical model a necklace and the spheres beads and we follow the same terminology. Deforming necklaces and efficient algorithms for this problem are needed in many computational fields including computer graphics, computer vision, robotics, geographic information systems, spatial databases, molecular biology, and scientific computing. There is a literature on using spheres in engineering modeling ${ }^{5}$. Computational problems involving motion can be stated in the Kinetic Data Structure Framework (KDS for short) ${ }^{9,8}$. A motion of necklaces models (i) the Brownian motion of molecules where necklaces move as rigid bodies, and (ii) molecular dynamics 
where necklaces only undergo local changes. Applied to deforming necklaces an efficient KDS can be designed using bounding volume hierarchies. Guibas et al. ${ }^{10}$ focused on a simple variant of hierarchy that uses spheres. They analyzed two ways of defining the spheres in the hierarchy. A spherical hierarchy of a necklace is defined to be a balanced tree whose leaves correspond to the beads. To each internal node there is an assigned cage that is a bounding sphere. A wrapped hierarchy is a spherical hierarchy of a necklace where the cage corresponding to each internal node is the minimum enclosing sphere of the beads in the canonical sub-necklace associated with that node. Another hierarchy called a layered hierarchy is defined by making the cage of an internal node to be the minimum enclosing sphere of the cages of its two children ${ }^{15}$.

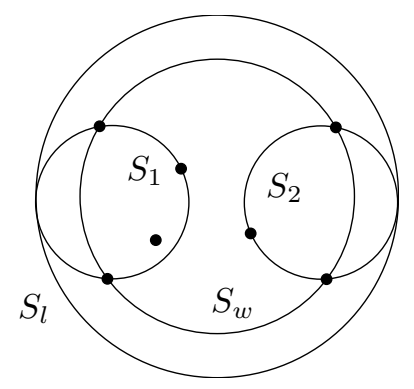

Fig. 1. $S_{w}$ is the wrapped sphere of 7 points and $S_{l}$ is the layered sphere constructed for the spheres $S_{1}$ and $S_{2}$.

The wrapped hierarchy is slightly more difficult to compute than the layered hierarchy although it is a tighter fitting, see Fig. 1 for an example and ${ }^{5}$ for detailed comparison of two hierarchies. The idea of using cylinders comes from this example. The spheres $S_{1}$ and $S_{2}$ can be covered by a cylinder of a volume smaller than the volume of $S_{l}$. The use of cylinders for molecular representation is not a new idea. The cylinders are widely used in software packages visualizing proteins. Structural components of proteins - helixes - are depicted by cylinders. For example, the human deoxyhaemoglobin $4 \mathrm{HHB}$ is a protein with high concentration of helices; it contains 32 helices. The helix structure is shown on Fig. 2. Packing helices into cylinders and related problems can be found in ${ }^{16}$.

We intend to represent a molecule using cylinders. Problems of covering spheres in $\mathbb{R}^{3}$ by cylinders are computationally difficult. Recently, Zhu ${ }^{18}$ considered the problem of covering points by cylinders. The problem is NP-hard even for points (spheres of radius 0 ). In this paper we address the following problem.

Necklace packing into cylinders. Let $\mathcal{N}$ be a necklace consisting of $n$ beads $B_{1}, B_{2}, \ldots, B_{n}$ in $\mathbb{R}^{3}$. Let $k$ be an integer $1 \leq k \leq n$. Find $k$ cylinders $C_{1}, C_{2}, \ldots, C_{k}$ and a partition of the necklace into 


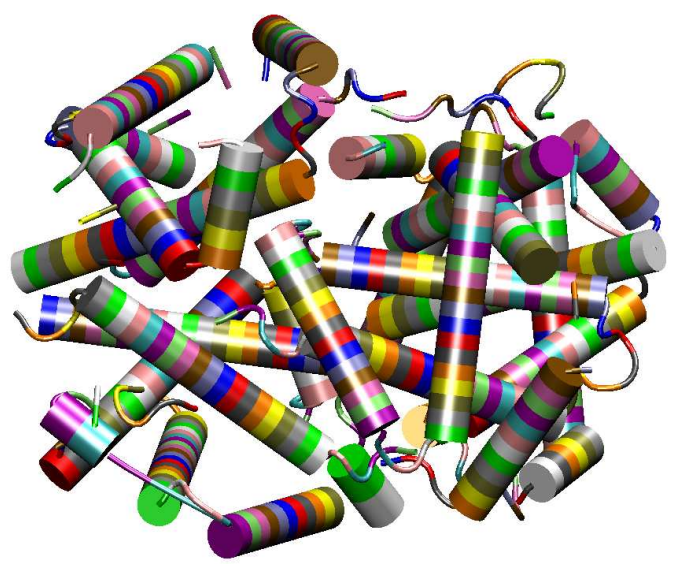

Fig. 2. The helices of human deoxyhaemoglobin 4HHB.

$k$ sub-necklaces $\mathcal{N}_{1}, \mathcal{N}_{2}, \ldots, \mathcal{N}_{k}$ such that, for each $1 \leq i \leq k$, the cylinder $C_{i}$ contains the beads of the necklace $\mathcal{N}_{i}$ and a function $F\left(C_{1}, C_{2}, \ldots, C_{k}\right)$ is minimized. Examples of function $F()$ can be (i) the sum of volumes of the cylinders, or (ii) the maximum radius of a cylinder, or (iii) the maximum volume of a cylinder, etc.

We show that, for reasonable functions $F$, the problem of necklace packing can be solved in polynomial time. The algorithm exploits the sequence property of necklaces and is in contrast to NP-hardness result of packing a set of distributed points ${ }^{18}$.

We use the necklace packing to generate a cylindrical hierarchy. As in the spherical hierarchy ${ }^{10}$, there are two options here: wrapped and layered hierarchy. We define a cylinder cage to be the smallest enclosing cylinder of underlying cylinders where a cylinder is measured using the function $F()$.

We also mention that the smallest radius cylinders find applications in the projective clustering ${ }^{2,12}$.

\section{Smallest Enclosing Cylinder}

Given a necklace $\mathcal{N}$, we want to find the smallest cylinder containing all the spheres of $\mathcal{N}$. Unfortunately, it seems that the property of spheres of being in the necklace does not help in computing the smallest enclosing cylinder. There are several results on computing a cylinder with the smallest radius ${ }^{1,4,17}$. Agarwal et al. ${ }^{1}$ gave a $O\left(n^{3+\delta}\right)$ time algorithm for any $\delta>0$. Schömer et al. ${ }^{17}$ found a $O\left(n \varepsilon^{-2} \log \varepsilon^{-1}\right)$ time algorithm that computes $(1+\varepsilon)$-approximation of the smallest radius for any $\varepsilon>0$. Chan ${ }^{4}$ improved the running time to $O(n / \varepsilon)$ using convex programming. Zhu ${ }^{18}$ obtained a practical algorithm with running time $O\left(n \log n+n / \varepsilon^{4}\right)$. We 
give a practical algorithm that computes $(1+\varepsilon)$-approximation of the smallest cylinder under various objective functions $F()$. We denote $i$-th bead in the necklace by $B_{i}\left(o_{i}, r_{i}\right)$ where $o_{i}$ is the center of $B_{i}$ and $r_{i}$ is its radius. The coordinates of a point $p \in \mathbb{R}^{3}$ are denoted by $(x(p), y(p), z(p))$ or $\left(p_{x}, p_{y}, p_{z}\right)$.

\section{Algorithm 1}

Step 1. Compute

$$
\mathcal{B}=\left[c_{x}-a_{x}, c_{x}+a_{x}\right] \times\left[c_{y}-a_{y}, c_{y}+a_{y}\right] \times\left[c_{z}-a_{z}, c_{z}+a_{z}\right],
$$

the bounding box of the spheres in the necklace $\mathcal{N}$. For example,

$$
c_{x}-a_{x}=\min _{1 \leq i \leq n}\left\{x\left(o_{i}\right)-r_{i}\right\} .
$$

Step 2. Let $r=\max \left(a_{x}, a_{y}, a_{z}\right)$ and let

$$
D=\left[c_{x}-r, c_{x}+r\right] \times\left[c_{y}-r, c_{y}+r\right] \times\left[c_{z}-r, c_{z}+r\right] .
$$

Step 3. Generate a grid of size $N \times N$ on each face of the cube $D$. Let $G$ be the set of all grid points.

Step 4. For each line $p_{1} p_{2}$ defined by two grid points $p_{1}, p_{2} \in G$, find the smallest cylinder $C$ with the centerline $p_{1} p_{2}$ that contains all the beads of the necklace. Compute $F(C)$.

Step 5. Compute the smallest value of $F(C)$ obtained in Step 4.

Theorem 1. Let $F(C)$ be one of the following functions: (1) the radius of the cylinder $C$, or $(2)$ the volume of the cylinder $C$. Algorithm 1 computes $(1+\varepsilon)$ approximation of the smallest enclosing cylinder in $O\left(n / \varepsilon^{2}\right)$ time using $N=$ $\lceil 1 /(2 \varepsilon)\rceil$ for both cases.

Proof. Let $C^{*}$ be the optimal cylinder and let $l^{*}$ be its centerline and let $r *$ be its radius. The line $l^{*}$ intersects the box $\mathcal{B}$; otherwise $l^{*}$ can be separated from $\mathcal{B}$ by a plane which means that $C^{*}$ is not optimal. The line $l^{*}$ intersects the boundary of $\mathcal{B}$ by two points, say $q_{1}$ and $q_{2}$. Let $p_{i}, i=1,2$ be the grid point closest to $q_{i}$ and let $C$ be the cylinder computed for the line $p_{1} p_{2}$. The angle $\alpha$ between the lines $l^{*}$ and $p_{1} p_{2}$ is bounded by $\arcsin (\varepsilon)$. Therefore the radius of the cylinder $C$ differs from $r^{*}$ by no more than $\varepsilon$. This implies the bound for the first case.

Consider the second case where the volume is minimized. Let $h^{*}$ be the height of the cylinder $C^{*}$. The height of $C$ is no longer than $h^{*} \cos \alpha=h^{*}\left(1+O\left(\varepsilon^{2}\right)\right)$. The volume of $C$ is at most $(1+O(\varepsilon)) V^{*}$ where $V^{*}$ is the volume of $C^{*}$. The theorem follows.

Remark. Algorithm 1 is practical. We implemented it to analyze the performance of a cylindrical hierarchy built on the top of Algorithm 1. A faster but more complicated algorithm (still simpler but asymptotically slower than the algorithms 
by Chan ${ }^{4}$ ) can be obtained by checking $O(1 / \varepsilon)$ directions and, for each direction, finding the minimum enclosing circle of the points projected onto the plane orthogonal to the direction.

The exact algorithm by Agarwal et al. ${ }^{1}$ can be applied to the minimization of the radius. What if one want to minimize the volume of a cylinder?

Theorem 2. The smallest volume cylinder enclosing a necklace of $n$ beads can be computed in $O\left(n^{6}\right)$ time.

Proof. The volume of a cylinder $C$ of radius $r$ and height $h$ is $\operatorname{vol}(C)=\pi r^{2} h$. Let $C^{*}$ be the smallest volume cylinder enclosing a necklace $\mathcal{N}$. We assume that the beads of $\mathcal{N}$ are in general position, i.e. there is no unbounded cylinder whose surface touches more than four beads. The surface of $C^{*}$ has three components: two disks $D_{1}$ and $D_{2}$ and the cylindrical surface $S$ that can be rolled out as a rectangle. Note that each disk $D_{1}$ and $D_{2}$ touches a bead since $C^{*}$ has the smallest volume. The cylinder $C^{*}$ has a property that either

(i) the surface $S$ touches four beads, or

(ii) the surface $S$ touches only three beads.

In the first case the centerline of $C^{*}$ is determined by four touching beads and two disks $D_{1}$ and $D_{2}$ are tangent to two extreme beads in the direction of the centerline. There are $O\left(n^{4}\right)$ possible ways to choose these beads. Each combination of beads generate $O(1)$ centerlines of a cylinder touching them simultaneously. We compute each of these lines and find two corresponding disks $D_{1}$ and $D_{2}$. This gives us the volume of the cylinder. We check in linear time if the cylinder contains all the beads of $\mathcal{N}$. Thus, the first case can be processed in $O\left(n^{5}\right)$ time.

In the second case the location of the cylinder in the space is determined by five beads: three beads $B_{1}, B_{2}, B_{3}$ touching $S$ and two beads $B_{4}, B_{5}$ touching the disks $D_{1}$ and $D_{2}$. Note that beads $B_{1}, B_{2}$ and $B_{3}$ are distinct but two sets $\left\{B_{1}, B_{2}, B_{3}\right\}$ and $\left\{B_{4}, B_{5}\right\}$ can intersect. There are $O\left(n^{5}\right)$ choices to select the beads. Each tuple generates $O(1)$ cylinders minimizing the volume. Each cylinder can be checked if it contains all the beads of $\mathcal{N}$. The running time in the second case is $O\left(n^{6}\right)$.

Next, we describe a more complicated algorithm with better asymptotic runtime.

Theorem 3. Using advanced techniques the smallest volume cylinder enclosing a necklace of $n$ beads can be computed in $O\left(n^{5+\delta}\right)$ time where $\delta>0$ is arbitrary small constant.

Proof. We apply the parametric search technique of Megiddo ${ }^{14}$. We consider the following decision problem.

Decision Problem. Given a necklace $\mathcal{N}$ and a parameter $V$, decide if there is a cylinder of volume $V$ that contains all the beads of $\mathcal{N}$. 
Let $L$ be a line in $\mathbb{R}^{3}$ and let $C$ be the smallest cylinder with the centerline $L$ containing the necklace. Let $\left(\xi_{1}, \xi_{2}, 1\right)$ be the direction of $L$, i.e. the line $L$ is parallel to the line $L\left(\xi_{1}, \xi_{2}\right)$ passing through the origin $O(0,0,0)$ and the point $\left(\xi_{1}, \xi_{2}, 1\right)$. Consider an $i$-th bead $B_{i}$. To simplify notation, let $o_{i}=\left(x_{i}, y_{i}, z_{i}\right)$. There are two planes, denoted by $\pi_{i}^{-}$and $\pi_{i}^{+}$, orthogonal to the direction $\left(\xi_{1}, \xi_{2}, 1\right)$ and tangent to the bead $B_{i}$. Their equations can be written as $\xi_{1} x+\xi_{2} y+z=h_{i}^{-}\left(\xi_{1}, \xi_{2}\right)$ and $\xi_{1} x+\xi_{2} y+z=h_{i}^{+}\left(\xi_{1}, \xi_{2}\right)$.

The values of $h_{i}^{-}\left(\xi_{1}, \xi_{2}\right)$ and $h_{i}^{+}\left(\xi_{1}, \xi_{2}\right)$ can be computed as follows. Let $o_{i}^{\prime}$ be the projection of the point $o_{i}$ to the line $L\left(\xi_{1}, \xi_{2}\right)$, see Fig. 3. Then the coordinates of $o_{i}^{\prime}$ satisfy $x\left(o_{i}^{\prime}\right)=\lambda_{i} \xi_{1}, y\left(o_{i}^{\prime}\right)=\lambda_{i} \xi_{2}$ and $z\left(o_{i}^{\prime}\right)=\lambda_{i}$ for some real number $\lambda_{i}$. The segment $o_{i} o_{i}^{\prime}$ is orthogonal to the segment $O o_{i}^{\prime}$. Thus

$$
\begin{gathered}
\lambda_{i} \xi_{1}\left(\lambda_{i} \xi_{1}-x_{i}\right)+\lambda_{i} \xi_{2}\left(\lambda_{i} \xi_{2}-y_{i}\right)+\lambda_{i}\left(\lambda_{i}-z_{i}\right)=0 \\
\lambda_{i}\left(\xi_{1}^{2}+\xi_{2}^{2}+1\right)=\xi_{1} x_{i}+\xi_{2} y_{i}+z_{i} .
\end{gathered}
$$

Let $\alpha=\xi_{1}^{2}+\xi_{2}^{2}+1$. Clearly, $\alpha>0$. The planes $\pi_{i}^{+}$and $\pi_{i}^{-}$intersect the line $L\left(\xi_{1}, \xi_{2}\right)$ at the points $H_{i}^{+}\left(\lambda_{i}^{+} \xi_{1}, \lambda_{i}^{+} \xi_{2}, \lambda_{i}^{+}\right)$and $H_{i}^{-}\left(\lambda_{i}^{-} \xi_{1}, \lambda_{i}^{-} \xi_{2}, \lambda_{i}^{-}\right)$, respectively, where $\lambda_{i}^{+}=\lambda_{i}+r_{i} / \alpha$ and $\lambda_{i}^{-}=\lambda_{i}-r_{i} / \alpha$, see Fig. 3 . The values of $h_{i}^{-}\left(\xi_{1}, \xi_{2}\right)$ and $h_{i}^{+}\left(\xi_{1}, \xi_{2}\right)$ can be obtained by substituting the points $H_{i}^{-}$and $H_{i}^{+}$in the equations of the planes $\pi_{i}^{-}$and $\pi_{i}^{+}$

$$
\begin{aligned}
& h_{i}^{-}\left(\xi_{1}, \xi_{2}\right)=\xi_{1} x_{i}+\xi_{2} y_{i}+z_{i}-r_{i} \\
& h_{i}^{+}\left(\xi_{1}, \xi_{2}\right)=\xi_{1} x_{i}+\xi_{2} y_{i}+z_{i}+r_{i} .
\end{aligned}
$$

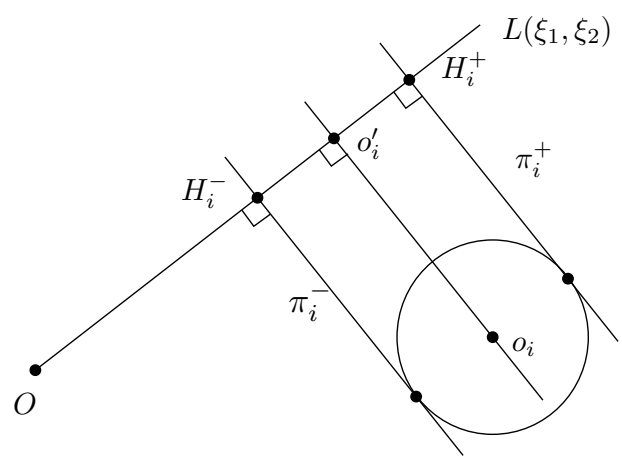

Fig. 3. The points $H_{i}^{-}$and $H_{i}^{+}$.

Suppose that $H_{i}^{+}$and $H_{j}^{-}$are the extreme points on the line $L\left(\xi_{i}, \xi_{2}\right)$ among all the points $H_{l}^{-}, H_{l}^{+}, l=1, \ldots, n$. Then the points $H_{i}^{+}$and $H_{j}^{-}$determine two disks of the cylinder $C$. The height of $C$ is

$$
h=\left|H_{i}^{+} H_{j}^{-}\right|=\sqrt{\left(\lambda_{i}^{+} \xi_{1}-\lambda_{j}^{-} \xi_{1}\right)^{2}+\left(\lambda_{i}^{+} \xi_{2}-\lambda_{j}^{-} \xi_{2}\right)^{2}+\left(\lambda_{i}^{+}-\lambda_{j}^{-}\right)^{2}}
$$




$$
=\left|\lambda_{i}^{+}-\lambda_{j}^{-}\right| \sqrt{\alpha}
$$

Note that $\lambda_{i}^{+} \geq \lambda_{j}^{-}$. Then

$$
\begin{aligned}
h & =\left(\lambda_{i}^{+}-\lambda_{j}^{-}\right) \sqrt{\alpha}=\left(\lambda_{i}+r_{i} / \alpha-\lambda_{j}-r_{j} / \alpha\right) \sqrt{\alpha} \\
& =\left(\xi_{1}\left(x_{i}-x_{j}\right)+\xi_{2}\left(y_{i}-y_{j}\right)+\left(z_{i}-z_{j}\right)+\left(r_{i}-r_{j}\right)\right) / \sqrt{\alpha} .
\end{aligned}
$$

Since the volume of the cylinder is $V=\pi r^{2} h$, the radius of the cylinder is $r=\sqrt{V /(\pi h)}$. The existence of the cylinder of radius $r$ containing the beads is related to transversals ${ }^{1}$. A line $L$ is called a (line) transversal of the necklace $\mathcal{N}$ if it intersects every bead of $\mathcal{N}$. Let $\mathcal{T}(\mathcal{N})$ denote the set of all transversals of $\mathcal{N}$. The reduction to transversals is as follows. The set of balls $B_{i}\left(o_{i}, r_{i}\right)$ can be wrapped by a cylinder of radius $r$ if and only if (i) $r_{i} \leq r$ for all $i$, and (ii) the balls $B_{i}^{\prime}\left(o_{i}, r-r_{i}\right)$ admit a transversal. For our purposes we need a constrained reduction: the set of balls $B_{i}\left(o_{i}, r_{i}\right)$ can be wrapped by a cylinder whose centerline is parallel to $L\left(\xi_{1}, \xi_{2}\right)$ and radius $r$ if and only if (i) $r_{i} \leq r$ for all $i$, and (ii) the balls $B_{i}^{\prime}\left(o_{i}, r-r_{i}\right)$ admit a transversal parallel to $L\left(\xi_{1}, \xi_{2}\right)$. Agarwal et al. ${ }^{1}$ solved the transversal detection problem using minimization/maximization diagrams of bivariate functions. We apply their approach to other functions since we use different parametrization.

A line $L^{\prime}$ parallel to the line $L\left(\xi_{1}, \xi_{2}\right)$ can be parameterized using two more variables $\xi_{3}$ and $\xi_{4}$ as follows:

$$
L^{\prime}\left(\xi_{1}, \xi_{2}, \xi_{3}, \xi_{4}\right)=\left\{\left(\xi_{3}+t \xi_{1}, \xi_{4}+t \xi_{2}, t\right) \mid t \in \mathbb{R}\right\} .
$$

Let $\pi^{\prime}\left(\xi_{1}, \xi_{2}, \xi_{3}\right)$ be the plane containing all the lines $L^{\prime}\left(\xi_{1}, \xi_{2}, \xi_{3}, \xi_{4}\right), \xi_{4} \in \mathbb{R}$. Consider an $l$-th bead $B_{l}^{\prime}$. We define two functions $f_{l}\left(\xi_{1}, \xi_{2}, \xi_{3}\right)$ and $g_{l}\left(\xi_{1}, \xi_{2}, \xi_{3}\right)$ as follows. Intersect $B_{l}^{\prime}$ with the plane $\tau_{l}$ passing through the center of $B_{l}^{\prime}$ and parallel to the $x z$-plane. The equation of the plane $\tau_{l}$ is $y=y_{l}$. Let $\sigma_{l}^{+}$and $\sigma_{l}^{-}$be two hemispheres defined by cutting $B_{l}^{\prime}$ by $\tau_{l}$. We assume that $\sigma_{l}^{+}$lies in the halfspace $y \geq y_{l}$ and $\sigma_{l}^{-}$lies in the halfspace $y \leq y_{l}$. If the plane $\pi^{\prime}\left(\xi_{1}, \xi_{2}, \xi_{3}\right)$ intersects the bead $B_{l}^{\prime}$, then there are two lines $L^{\prime}\left(\xi_{1}, \xi_{2}, \xi_{3}, a\right)$ and $L^{\prime}\left(\xi_{1}, \xi_{2}, \xi_{3}, b\right), a \leq b$ tangent to the hemispheres $\sigma_{l}^{-}$and $\sigma_{l}^{+}$, respectively. We define $g_{l}\left(\xi_{1}, \xi_{2}, \xi_{3}\right)=a$ and $f_{l}\left(\xi_{1}, \xi_{2}, \xi_{3}\right)=b$. In the second case $\pi^{\prime}\left(\xi_{1}, \xi_{2}, \xi_{3}\right) \cap B_{l}=\emptyset$ we define $f_{l}\left(\xi_{1}, \xi_{2}, \xi_{3}\right)=+\infty$ and $g_{l}\left(\xi_{1}, \xi_{2}, \xi_{3}\right)=-\infty$. The functions $f_{l}$ and $g_{l}$ have property that the line $L^{\prime}\left(\xi_{1}, \xi_{2}, \xi_{3}, \xi_{4}\right)$ intersects the bead $B_{l}^{\prime}$ if and only if $f_{l}\left(\xi_{1}, \xi_{2}, \xi_{3}\right) \leq \xi_{4} \leq g_{l}\left(\xi_{1}, \xi_{2}, \xi_{3}\right)$. The line $L^{\prime}\left(\xi_{1}, \xi_{2}, \xi_{3}, \xi_{4}\right)$ is transversal of the beads if and only if

$$
\max _{1 \leq l \leq n} f_{l}\left(\xi_{1}, \xi_{2}, \xi_{3}\right) \leq \xi_{4} \leq \min _{1 \leq l \leq n} g_{l}\left(\xi_{1}, \xi_{2}, \xi_{3}\right) .
$$

We show that the functions $f_{l}\left(\xi_{1}, \xi_{2}, \xi_{3}\right)$ and $g_{l}\left(\xi_{1}, \xi_{2}, \xi_{3}\right)$ have constant description complexity ${ }^{1}$, that is, the graph of each function is a semi-algebraic set in $\mathbb{R}^{4}$ defined by a constant number of polynomial equalities and inequalities of constant 
degree. We assume that $i$ and $j$ are fixed. By Equation (3) the radius of the cylinder satisfies

$$
r^{2}\left(\xi_{1}^{2}+\xi_{2}^{2}+1\right)=\left(\xi_{1}\left(x_{i}-x_{j}\right)+\xi_{2}\left(y_{i}-y_{j}\right)+\left(z_{i}-z_{j}\right)+\left(r_{i}-r_{j}\right)\right)^{2} .
$$

The lines tangent to the hemispheres $\sigma_{l}^{-}$and $\sigma_{l}^{+}$are at distance $r-r_{l}$ from the center $o_{l}$. This is equivalent to the condition that the line $L\left(\xi_{1}, \xi_{2}\right)$ is at distance $r-r_{l}$ from the point $p_{l}\left(x_{l}^{\prime}, y_{l}^{\prime}, z_{l}^{\prime}\right)$ where $x_{l}^{\prime}=x_{l}-\xi_{3}, y_{l}^{\prime}=y_{l}-\xi_{4}, z_{l}^{\prime}=z_{l}$. Substituting $i$ by $l$ and $o_{i}$ by $p_{l}$ in Equation (1) the nearest point on $L\left(\xi_{1}, \xi_{2}\right)$ to $p_{l}$ has coordinates $\left(\lambda \xi_{1}, \lambda \xi_{2}, \lambda\right)$ where

$$
\lambda=\left(\xi_{1} x_{l}^{\prime}+\xi_{2} y_{l}^{\prime}+z_{l}^{\prime}\right) /\left(\xi_{1}^{2}+\xi_{2}^{2}+1\right) .
$$

Therefore the tangent lines satisfy

$$
\begin{aligned}
\left(r-r_{l}\right)^{2} & =\left(\lambda \xi_{1}-x_{l}^{\prime}\right)^{2}+\left(\lambda \xi_{2}-y_{l}^{\prime}\right)^{2}+\left(\lambda-z_{l}^{\prime}\right)^{2} \\
& =\lambda^{2} \alpha-2 \lambda\left(\xi_{1} x_{l}^{\prime}+\xi_{2} y_{l}^{\prime}+z_{l}^{\prime}\right)+\left(x_{l}^{\prime}\right)^{2}+\left(y_{l}^{\prime}\right)^{2}+\left(z_{l}^{\prime}\right)^{2} \\
& =\left(x_{l}^{\prime}\right)^{2}+\left(y_{l}^{\prime}\right)^{2}+\left(z_{l}^{\prime}\right)^{2}-\lambda^{2} \alpha .
\end{aligned}
$$

Plugging $\lambda$ from (5) and $x_{l}^{\prime}, y_{l}^{\prime}, z_{l}^{\prime}$ into (6) we obtain a polynomial of a constant degree.

Recently Koltun and Sharir ${ }^{13}$ proved that the overlay of two trivariate diagrams has $O\left(n^{3+\delta}\right)$ complexity. Applied to the maximization diagram of $f_{l}\left(\xi_{1}, \xi_{2}, \xi_{3}\right)$ and the minimization diagram of $g_{l}\left(\xi_{1}, \xi_{2}, \xi_{3}\right)$ we obtain $O\left(n^{3+\delta}\right)$ bound for the fixed pair $(i, j)$. The decision problem can be solved in $O\left(n^{5+\delta}\right)$ time since there are $O\left(n^{2}\right)$ pairs $(i, j)$. The parametric search technique allows us to solve the optimization problem within the same bound $O\left(n^{5+\delta}\right)$.

\section{General $k$}

In this Section we show how to find an optimal necklace packing into cylinders. Our algorithm is based on the dynamic programming approach. Essentially, a polynomial time algorithm is possible to design since the problem is decomposable into polynomially many subproblems.

Theorem 4. Let $F(C)$ be one of the following functions: (1) the radius of the cylinder $C$, or (2) the volume of the cylinder $C$. Let $F\left(C_{1}, \ldots, C_{k}\right)$ is defined to be either minmax or minsum of the values of $F\left(C_{1}\right), \ldots, F\left(C_{k}\right)$. The problem of necklace packing into cylinders minimizing $F()$ can be solved in $O\left(n^{5+\delta}\right)$ time for the case (1) and in $O\left(n^{7+\delta}\right)$ time for the case (2).

Proof. Let $i$ and $j$ be two integers such that $1 \leq i \leq j \leq n$. Let $\mathcal{N}(i, j)$ denote the sub-necklace of $\mathcal{N}$ with beads $B_{i}, B_{i+1}, \ldots, B_{j}$. Let $C(i, j)$ be the optimal cylinder covering the necklace $\mathcal{N}(i, j)$. By Theorem 3 the cylinder $C(i, j)$ can be found in $O\left(n^{5+\delta}\right)$ time if $F(C)$ is the volume function. Let $\Xi(i, j)$ denote the value of 
$F(C(i, j))$. Therefore the values of $\Xi(i, j)$ for all $i$ and $j$ can be computed in $O\left(n^{7+\delta}\right)$ time. The bound for the first case follows if we apply the $O\left(n^{3+\delta}\right)$-algorithm by Agarwal et al. ${ }^{1}$.

Let $m$ be an integer $1 \leq m \leq k$. Let $\mathcal{C}(j, m)=\left\{C_{1}, C_{2}, \ldots, C_{m}\right\}$ be the set of $m$ cylinders in the optimal packing of the necklace $\mathcal{N}(1, j)$ with $m$ cylinders. Let $\Phi(j, m)$ denote the value of $F\left(C_{1}, C_{2}, \ldots, C_{m}\right)$. Then

$$
\begin{aligned}
\Phi(j, 1) & =\Xi(1, j) \quad \text { for all } 1 \leq j \leq n \\
\Phi(j, m) & =\min _{1 \leq i<j}\{\Phi(i, m-1)+\Xi(i+1, j)\} \quad \text { for all } 1 \leq j \leq n \text { and } 1 \leq m \leq k
\end{aligned}
$$

The dynamic program computes the values of $\Phi(j, m)$ using the above equations. This computation takes $O\left(n^{2}\right)$ time. Clearly, the optimal value of the necklace packing is $\Phi(n, k)$. The theorem follows.

We remark that the approach based on dynamic programming can be applied to many other objective functions that are decomposable.

\section{Cylindrical Hierarchy}

We consider two hierarchies wrapped and layered. A cage of a node in the wrapped hierarchy is defined as the optimal cylinder covering all beads in the corresponding subtree. In the layered hierarchy the cage is the smallest cylinder containing the cages of its children. At first glance, computation of the wrapped hierarchy is more difficult than computation of one cylinder. We show that the computing time is the same for the exact problem and slightly bigger for the approximate one.

\subsection{Wrapped Hierarchy}

Lemma 1. The wrapped hierarchy can be constructed in

(a) $O\left(n^{3+\delta}\right)$ time exactly if the objective function is based on the radius only,

(b) $O\left(n^{5+\delta}\right)$ time exactly if the objective function is based on the volume,

(c) $O\left((n \log n) / \varepsilon^{4}\right)$ time approximately if the objective function is based either on the radius or the volume.

Proof. The main computational task is to construct all the cages of the hierarchy. We construct each cage independently using the algorithms from Theorems 1 and 2 . The algorithm is recursive. Let $t(n)$ be the running time for computing the optimal cylinder (or its approximation) for $n$ spheres. Let $T(n)$ be the running time to construct the hierarchy for $n$ beads. Then

$$
T(1)=\text { const, and } T(n)=t(n)+2 T(n / 2) \text { if } n \geq 2 .
$$

The lemma follows since (a) $t(n)=n^{3+\delta}$, (b) $t(n)=n^{5+\delta}$, and (c) $t(n)=$ $O\left(n / \varepsilon^{4}\right)$. 


\subsection{Layered Hierarchy}

In the layered hierarchy we have a new problem.

Cage Problem. Let $C_{1}$ and $C_{2}$ be two cylinders in $\mathbb{R}^{3}$. Find an optimal cylinder $C$ that contains $C_{1}$ and $C_{2}$ where the quality of a cylinder is measured by a function $F()$ as in the necklace packing problem.

Lemma 2. The optimal cage can be found in $O(1)$ time. Thus the layered hierarchy can be constructed in $O(n)$ time.

Proof. We note that it is sufficient to substitute the cylinders $C_{1}$ and $C_{2}$ by four circles on their boundaries, see Fig. 4.
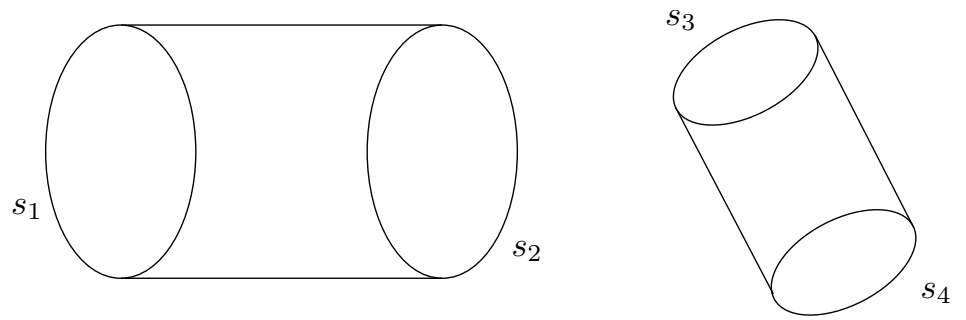

Fig. 4. The four circles $s_{1}, s_{2}, s_{3}$ and $s_{4}$ on the boundary of two cylinders.

The problem of finding an optimal cylinder has a constant complexity. We parameterize the cylinder using six variables. Let $a=\left(a_{x}, a_{y}, 0\right)$ and $b=\left(a_{x}+b_{x}, a_{y}+\right.$ $\left.b_{y}, 1\right)$ be two points that are the intersections of the centerline of the cylinder and the planes $z=0$ and $z=1$. We can assume that the centerline of the optimal cylinder is not parallel to the plane $O X Y$ by the perturbation argument. Two disks on the boundary of the cylinder can be parameterized as planes $b_{x} x+b_{y} y+z=h_{1}$ and $b_{x} x+b_{y} y+z=h_{2}$. The volume of the cylinder can be expressed using the variables $a_{x}, a_{y}, b_{x}, b_{y}, h_{1}, h_{2}$. The optimal value of the volume can be found in $O(1)$ time.

The total running time is linear since the recurrence for the running time is $T(n)=O(1)+2 T(n / 2)$.

\section{Experiments}

We have implemented Algorithm 1 for finding the smallest cylinder. A close-up of the atoms of a helix of human deoxyhaemoglobin $4 \mathrm{HHB}$ and the smallest enclosing cylinder is shown in Fig. 5. It demonstrates that the cylinders are useful for shape representation of molecules.

The performance of a cylindrical hierarchy can be tested by running real life applications like the collision detection. We implemented the cylindrical hierarchy 


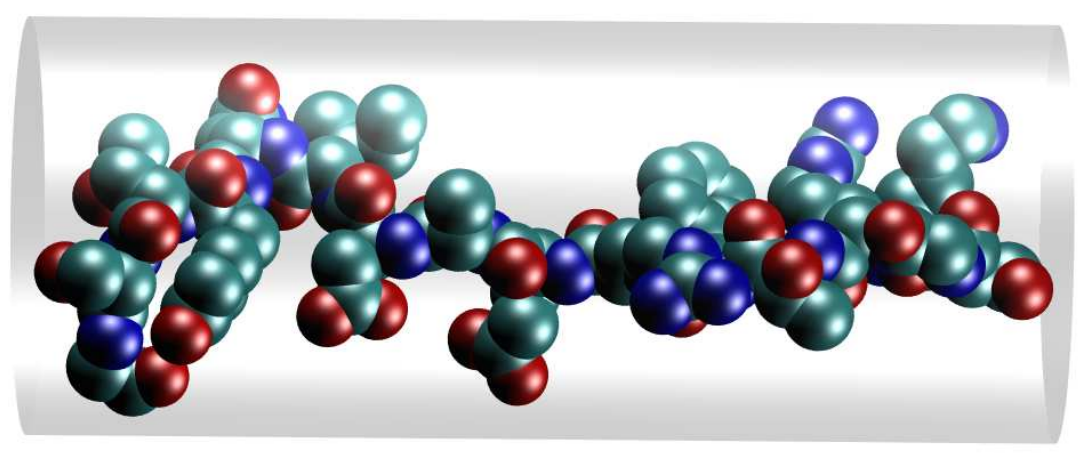

Fig. 5. The helix of human deoxyhaemoglobin $4 \mathrm{HHB}$ and its smallest enclosing cylinder.

based on the binary tree representation of the necklace. We select the wrapped hierarchy and the objective function minimizing the radius of a cylinder. We modify slightly the shape of the enclosing cylinders and consider cigars since they admit a simpler algorithm for detecting whether two cigars intersect. For two points $a, b \in$ $\mathbb{R}^{3}$ and a positive real number $r$, the cigar $\pi(a, b, r)$ is the Minkowski sum of the segment $[a, b]$ and the ball of radius $r$ centered at the origin, i.e.

$$
\pi(a, b, r)=\left\{p \in \mathbb{R}^{3}|\exists q \in[a, b],| p q \mid \leq r\right\}
$$

\subsection{The smallest cigar with a given centerline.}

Let $p_{1}$ and $p_{2}$ be two points and let $r$ be the smallest radius of a cylinder with the centerline $p_{1} p_{2}$ containing the necklace $\mathcal{N}$. We want to find the smallest cigar $\pi(a, b, r)$ containing the necklace $\mathcal{N}$ such that the segment $[a, b]$ lies on the line $p_{1} p_{2}$. For each bead $B_{i}$, we find two points $q_{i}^{+}$and $q_{i}^{-}$on the line $p_{1} p_{2}$ that are at distance $r-r_{i}$ from the bead center $o_{i}$ where the vector $q_{i}^{-} q_{i}^{+}$has the same direction as the vector $p_{1} p_{2}$, see Fig. 6 .

We assume that the distance between $p_{1}$ and $p_{2}$ is at least some positive constant as in Algorithm 1. Let $v$ be the unit vector $p_{2} p_{1} /\left|p_{2} p_{1}\right|$. The point $q_{i}$ of the line $p_{1} p_{2}$ closest to $o_{i}$ satisfies $q_{i}=p_{1}+\alpha_{i} v$ for some value of $\alpha_{i} \in \mathbb{R}$. Then the vectors $o_{i} q_{i}$ and $v$ are perpendicular. Thus $v \cdot o_{i} q_{i}=0$ or $v \cdot\left(\alpha_{i} v+p_{1} o_{i}\right)=0$ or $\alpha_{i}=v \cdot\left(o_{i} p_{1}\right)$. Let $d_{i}$ be the distance between $o_{i}$ and $q_{i}$. The distance from the points $q_{i}^{+}$and $q_{i}^{-}$ to $o_{i}$ is $r$. Therefore $q_{i}^{+}=q_{i}+\beta_{i} v$ and $q_{i}^{-}=q_{i}-\beta_{i} v$ where $\beta_{i}=\sqrt{r^{2}-\left|o_{i} q_{i}\right|^{2}}$. Let $\alpha_{i}+\beta_{i}$ be the minimum value among all $\alpha_{m}+\beta_{m}, 1 \leq m \leq n$ and let $\alpha_{j}-\beta_{j}$ be the maximum value among all $\alpha_{m}-\beta_{m}, 1 \leq m \leq n$. The segment of the optimal cigar is then $\left[p_{1}+\left(\alpha_{i}+\beta_{i}\right) v, p_{1}+\left(\alpha_{j}-\beta_{j}\right) v\right]$, see Fig. 6 . 


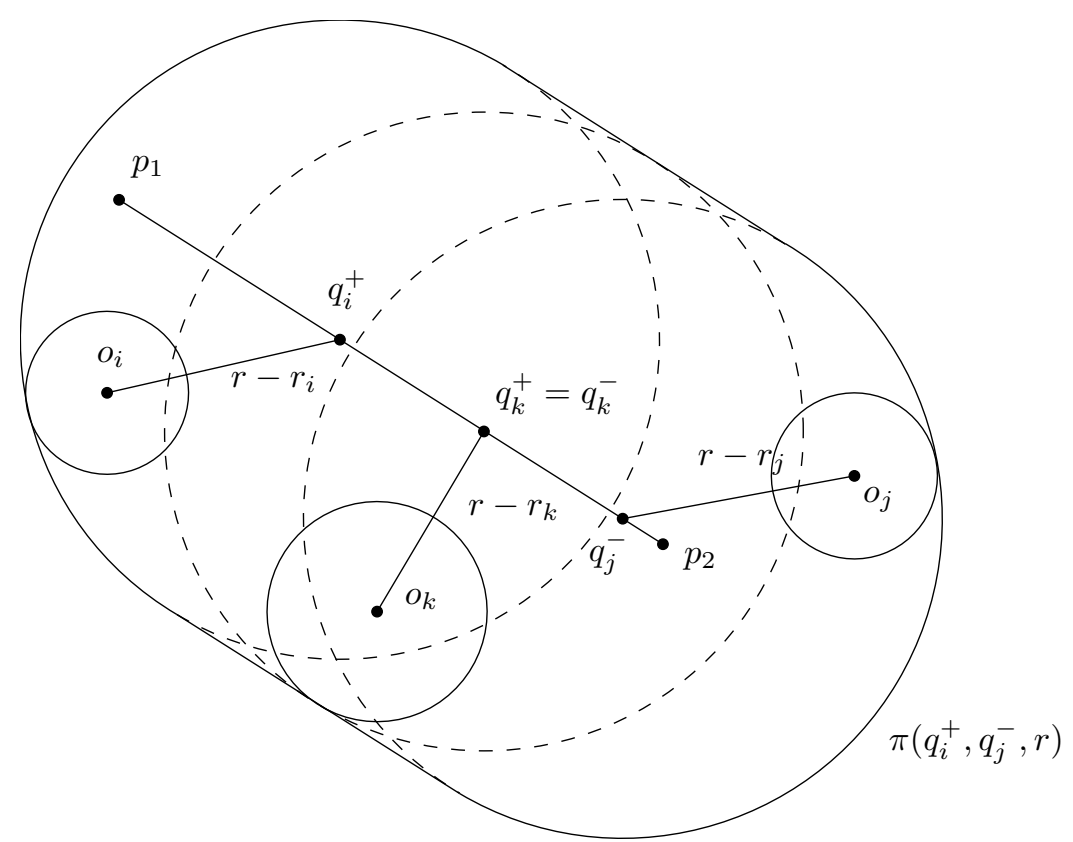

Fig. 6. The smallest cigar $\pi\left(q_{i}^{+}, q_{j}^{-}, r\right)$.

\subsection{Collision test}

In this section we show that it is not difficult to detect the intersection of cigars.

Lemma 3. Let $\pi_{1}\left(a_{1}, b_{1}, r_{1}\right)$ and $\pi_{2}\left(a_{2}, b_{2}, r_{2}\right)$ be two cigars. Let $d$ be the smallest distance between two straight line segments $\left[a_{1}, b_{1}\right]$ and $\left[a_{2}, b_{2}\right]$. Two cigars $\pi_{1}$ and $\pi_{2}$ intersect if and only if $d \leq r_{1}+r_{2}$.

Proof. Suppose that two cigars intersect and a point $p$ lies in the intersection of the cigars. There are points $p_{1} \in \pi_{1}$ and $p_{2} \in \pi_{2}$ such that the distances $\left|p p_{1}\right|$ and $\left|p p_{2}\right|$ are at most $r_{1}$ and $r_{2}$, respectively. Then $\left|p_{1} p_{2}\right| \leq p_{1} p|+| p p_{2} \mid \leq r_{1}+r_{2}$. The reverse claim can be shown by a similar argument.

The computation of the distance between two segments in $\mathbb{R}^{3}$ is the well-known problem due to applications in graphics and animations. The collision detection can be done using the following primitives:

- test whether two balls intersect,

- test whether a segment and a ball intersect,

- find the smallest distance between two lines.

Specifically, two cigars $\pi_{1}\left(a_{1}, b_{1}, r_{1}\right)$ and $\pi_{2}\left(a_{2}, b_{2}, r_{2}\right)$ intersect if and only if one of the following conditions holds: 
(i) Two balls in at least one pair $\left(B\left(a_{1}, r_{1}\right), B\left(a_{2}, r_{2}\right)\right),\left(B\left(a_{1}, r_{1}\right), B\left(b_{2}, r_{2}\right)\right)$, $\left(B\left(b_{1}, r_{1}\right), B\left(a_{2}, r_{2}\right)\right)$, or $\left(B\left(b_{1}, r_{1}\right), B\left(b_{2}, r_{2}\right)\right)$ intersect,

(ii) The perpendicular dropped from an endpoint $e$ of one segment $s \in$ $\left\{\left[a_{1}, b_{1}\right],\left[a_{2}, b_{2}\right]\right\}$ to the line $l$ containing the other segment $s^{\prime}$ meets a point $e^{\prime}$ of $s^{\prime}$ and $\left|e e^{\prime}\right| \leq r_{1}+r_{2}$.

(iii) Let $p_{1} p_{2}$ be the segment making the shortest distance between two lines containing $\left[a_{1}, b_{1}\right]$ and $\left[a_{2}, b_{2}\right]$, respectively. The distance $\left|p_{1} p_{2}\right|$ is at most $r_{1}+r_{2}$ and $p_{i} \in\left[a_{i}, b_{i}\right], i=1,2$.

\subsection{Collision detection}

Let $A$ and $B$ be two molecules and let $H_{A}$ and $H_{B}$ be their cylindrical hierarchies. We apply the following algorithm to detect if the necklaces of $A$ and $B$ intersect. Let $C_{A}$ and $C_{B}$ be the root cylinders of $H_{A}$ and $H_{B}$, respectively. If $C_{A} \cap C_{B}=\emptyset$ then $A$ and $B$ do not intersect. Otherwise we pick a cylinder with larger radius, say $C_{A}$. We check the cylinders of the root children of $H_{A}$ against $C_{B}$. In general, we have a list $L$ of pairs of cylinders $\left(C_{1}, C_{1}^{\prime}\right),\left(C_{2}, C_{2}^{\prime}\right), \ldots$ such that the cylinders in each pair intersect. We select a cylinder with maximum radius among all cylinders in the list, say $C_{1}$. Let $v$ be the node of $H_{A}$ storing $C_{1}$ and let $u$ be the node of $H_{B}$ storing $C_{1}^{\prime}$. Let $\bar{C}_{1}$ and $\bar{C}_{2}$ be the cylinders of the children of $v$. We replace the pair $\left(C_{1}, C_{1}^{\prime}\right)$ in the list by the pairs $\left(\bar{C}_{1}, C_{1}^{\prime}\right)$ and $\left(\bar{C}_{2}, C_{1}^{\prime}\right)$. If $v$ is a leaf of $H_{A}$ then we consider the children of $u$. If both $v$ and $u$ are leaves then the collision is detected and the algorithm stops; otherwise we repeat this process until $L$ is not empty.

We select the protein endonuclease barnase 1BRS.pdb for collision detection. The chains $\mathrm{A}$ and $\mathrm{D}$ of the protein are in a docking position where many atoms from A and D are close to each other but do not overlap. It can be viewed as a good benchmark for collision detection in proteins. It contains 864 atoms in the chain A and 691 atoms in the chain D. We count the total number of pairs of cylinders checked by the algorithm. To compare the performance of the cylindrical hierarchy we also implemented the spherical hierarchy and the collision detection similar to the algorithm above (the only difference is that we compare the radii of two spheres when new pairs are generated). We run both algorithms on 1BRS: the spherical hierarchy uses 3677 intersection tests and the cylindrical hierarchy uses 2195 intersection tests only.

\section{Conclusion}

We studied the problem of necklace packing into cylinders, the problem of constructing cylindrical hierarchies, and the problem of collision detection using cylindrical hierarchies. We presented efficient algorithms, approximate and exact, for constructing cylindrical hierarchies under different criteria of optimal cylinders. The approximate algorithm is practical and has been implemented. The experiments demonstrate that the cylindrical hierarchy is efficient for collision detection in proteins. 


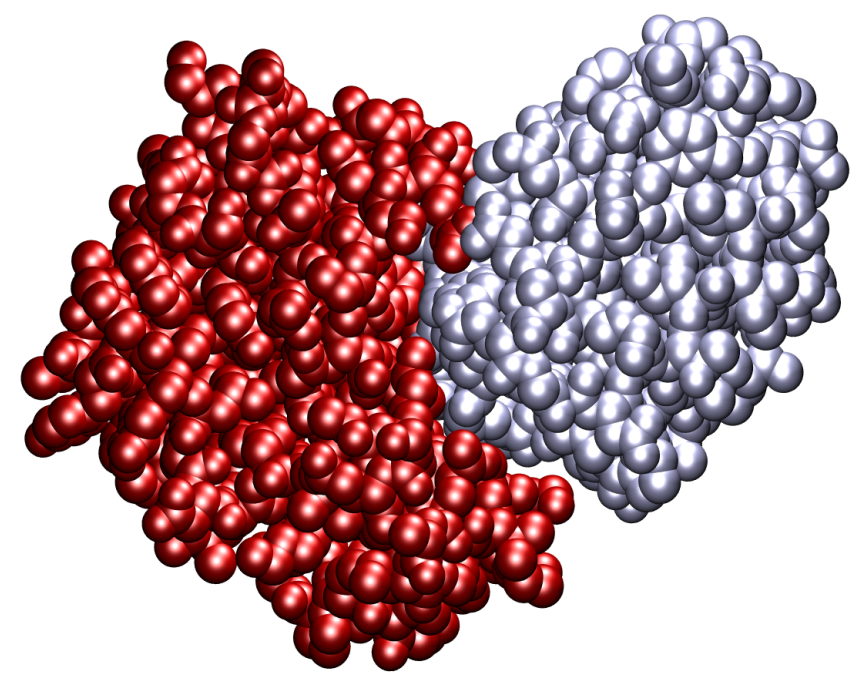

Fig. 7. Two chains of 1BRS.

Acknowledgments. I would like to thank the anonymous referees for their valuable comments.

\section{References}

1. P. K. Agarwal, B. Aronov, and M. Sharir. Line traversals of balls and smallest enclosing cylinders in three dimensions. Discrete Comput. Geom., 21:373-388, 1999.

2. P. K. Agarwal and C. M. Procopiuc. Approximation algorithms for projective clustering. In Proc. 11th ACM-SIAM Sympos. Discrete Algorithms, pp. 538-547, 2000.

3. S. Bespamyatnikh. Cylindrical hierarchy for deforming necklaces. In Proc. 9th Ann. Internat. Conf. Computing and Combinatorics, LNCS 2697, pp. 20-29.

4. T. Chan. Approximating the diameter, width, smallest enclosing cylinder, and minimum-width annulus. Int. J. Comput. Geom. Appl., 12(1-2):67-85, 2002.

5. S. De and K. J. Bathe. The method of finite spheres. Computational Mechanics, 25:329-345, 2000, http://link.springer-ny.com/link/service/journals/00466/ bibs/0025004/00250329.htm.

6. O. Devillers and F. P. Preparata. Culling a set of points for roundness or cylindricity evaluations. Int. J. Comput. Geom. Appl., 13(3):231-240, 2003.

7. K. Fischer and B. Gärtner. The smallest enclosing ball of balls - combinatorial structure and algorithms. In Proc. 19th Annu. ACM Sympos. Comput. Geom., pp. 292-301, 2003.

8. L. Guibas, F. Xie, and L. Zhang. Kinetic data structures for efficient simulation. In Proceedings of IEEE International Conference on Robotics and Automation, volume 3, pp. 2903-2910, 2001.

9. L. J. Guibas. Kinetic data structures - a state of the art report. In P. K. Agarwal, 
L. E. Kavraki, and M. Mason, editors, Proc. Workshop Algorithmic Found. Robot., pp. 191-209. A. K. Peters, Wellesley, MA, 1998.

10. L. J. Guibas, A. Nguyen, D. Russel, and L. Zhang. Collision detection for deforming necklaces. In Proc. 18th Annu. ACM Sympos. Comput. Geom., pp. 33-42, 2002.

11. D. Halperin and M. Overmars. Spheres, molecules, and hidden surface removal. Comput. Geom. Theory Appl., 11(2):83-102, 1998.

12. S. Har-Peled and K. Varadarajan. Projective clustering in high dimensions using coresets. In Proc. 18th Annu. ACM Sympos. Comput. Geom., pp. 312-318.

13. V. Koltun and M. Sharir. The partition technique for overlays of envelopes. In Proc. 43nd Annu. IEEE Sympos. Found. Comput. Sci., pp. 637-646.

14. N. Megiddo. Applying parallel computation algorithms in the design of serial algorithms. J. ACM, 30(4):852-865, 1983.

15. S. Quinlan. Efficient distance computation between non-convex objects. pp. 33243329, 1994.

16. J. Sadoc and N. Rivier. Boerdijk-coxeter helix and biological helices. The European Physical Journal B, 12(2):309-318, 1999.

17. E. Schömer, J. Sellen, M. Teichmann, and C. K. Yap. Smallest enclosing cylinders. Algorithmica, 27(2):170-186, 2000.

18. B. Zhu. Approximating 3D points with cylindrical segments. In Proc. 8th Ann. Internat. Conf. Computing and Combinatorics, pp. 420-429, 2002. 